\title{
Clear Cell Adenocarcinoma Originated from Adenomyosis
}

\author{
Sunwha Park, Angela Cho, Chul Min Park ${ }^{1}$ \\ Department of Obstetrics and Gynecology, Ewha Womans University Mokdong Hospital, Seoul, ${ }^{1}$ Department of Obstetrics and \\ Gynecology, Jeju National University Hospital, Jeju, Korea
}

\begin{abstract}
A 66-year-old postmenopausal woman received routine gynecologic check-up. Transvaginal ultrasonography and abdominal and pelvic computed tomography showed about 5-cm cystic mass in uterus with solid component and the patient had thin endometrium and the serum level of CA 125 was normal. We performed a total hysterectomy and bilateral salpingo-oophorectomy and found tumor which had brownish cystic fluid and about $2 \mathrm{~cm}$ sized and colored in light yellowish, polypoid protruding solid mass, located within the myometrial wall. Histopathological examination of frozen section revealed malignancy. The tumor was confined within the myometrium and its histologic type was clear cell adenocarcinoma. Finally we identified that the myometrial mass was clear cell adenocarcinoma originated from adenomyosis pathologically. The malignant transformation of adenomyosis is very rare. When we find a cystic change with solid component in adenomyosis patients, clear cell adenocarcinoma should be suspected as a differential diagnosis and magnetic resonance imaging should be considered for further evaluation. (Ewha Med J 2019;42(1):10-13)
\end{abstract}

\author{
Received July 16, 2018 \\ Revised December 28, 2018 \\ Accepted January 14, 2019 \\ Corresponding author \\ Chul Min Park \\ Department of Obstetrics and Gynecology, Jeju \\ National University Hospital, 15 Aran 13-gil, \\ Jeju 63241, Korea \\ Tel: 82-64-717-1823, Fax: 82-64-717-1131 \\ E-mail: obgymd@gmail.com
}

Key Words

Adenocarcinoma, clear cell; Adenomyosis; Myometrium

\section{Introduction}

The malignant transformation of patients with ovarian endometriosis and adenomyosis is very rarely encountered. Endometriosis is the rare origin of uterine cancers, which are seen fewer than $1 \%$ of all women with endometriosis [1]. At present, about 40 cases have been reported in English published literature [2]. Above all, clear cell adenocarcinoma (CCA) arising from adenomyosis has been exceedingly rarely reported $[2,3]$. Endometrioid adenocarcinoma is the most frequent histologic type of adenocarcinoma arising in endometriosis, followed by CCA [4]. Therefore, we report a very rare case of CCA arising from adenomyosis that could have been misdiagnosed or diagnosed in delay.

\section{Case}

A 66-year-old postmenopausal gravida 2 and para 2 woman received routine gynecologic check-up. She had no specific symptom and her medical history was not so special. Transvaginal ultrasonography showed about $5-\mathrm{cm}$ cystic mass in uterus with solid component and the patient had thin endometrium (Fig. 1). The serum level of CA 125 was $19.3 \mathrm{U} / \mathrm{mL}$ within normal range. Abdomen and pelvis $\mathrm{CT}$ revealed a $4.4-\mathrm{cm}^{-} \mathrm{cys}^{-}$ tic degenerative mass with enhancing solid portion consistent with myoma in the anterior portion of uterine body. But differential diagnosis for endometrial stromal sarcoma or leiomyosarcoma was required. The cystic degenerative mass was located in myometrium, maybe not involved endometrium and no tumor was detected in other organs including lymph node (Fig. 2).

Preoperative differential diagnoses included myoma, leiomyo-

This is an Open Access article distributed under the terms of the Creative Commons Attribution Non-Commercial License (http://creativecommons.org/licenses/by-nc/4.0) which permits unrestricted non-commercial use, distribution, and reproduction in any medium, provided the original work is properly cited. 
sarcoma or endometrial stromal sarcoma. We performed a total laparoscopic hysterectomy and bilateral salpingo-oophorectomy. And then the uterus and both adnexaes were extracted through the vaginal opening with intact state. We opened uterus and found tumor which had brownish cystic fluid and about $2 \mathrm{~cm}$ sized and colored in light yellowish, polypoid protruding solid mass. The mass was located only within the myometrial wall. Histopathological examination of frozen section revealed malignancy. So, staging operation including pelvic lymphadenectomy, paraaortic lymphadenectomy and appendectomy was performed additionally. During operation, there was no evidence of gross dissemination.

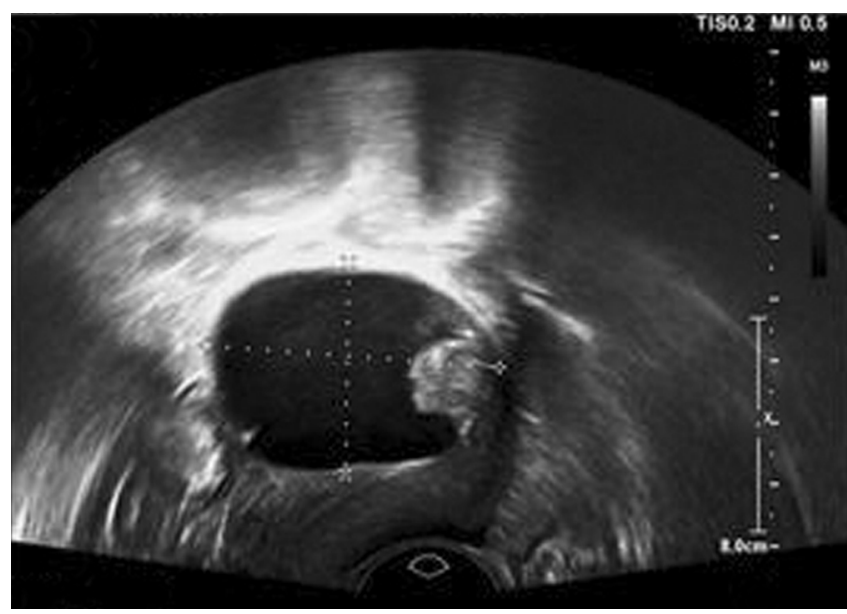

Fig. 1. Trans-vaginal ultrasonography showed 5 -cm cystic mass in uterus with solid component.

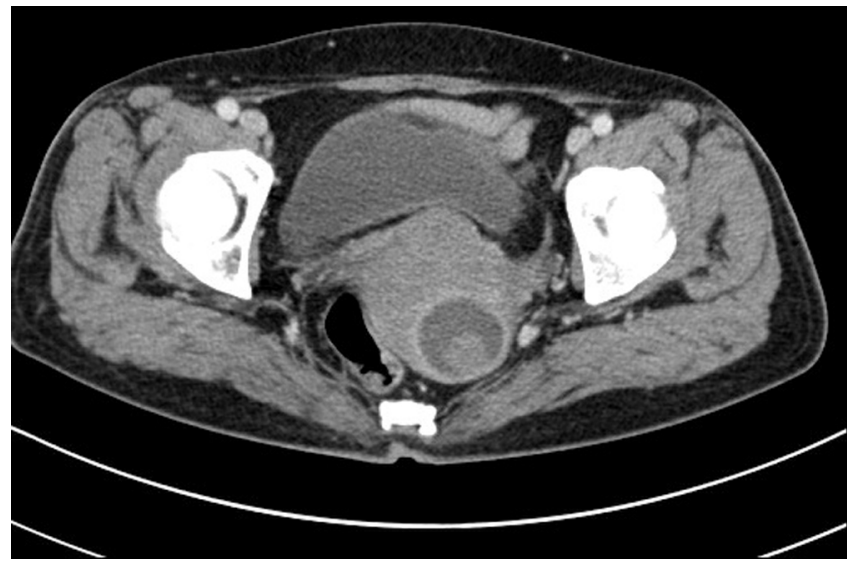

Fig. 2. Abdomen pelvic CT revealed a 4.4-cm cystic degenerative mass with enhancing solid portion consistent with myoma in the anterior portion of uterine body. The cystic degenerative mass was located in myometrium, maybe not involved endometrium.
Grossly, the uterus showed an irregularly-shaped, solid tumor mass $\left(2 \times 1.5 \times 1.5 \mathrm{~cm}^{3}\right)$ surrounded by smooth membranous wall. When we opened the specimen, the endometrial canal was well preserved and covered by atrophic endometrial tissue. Several adenomyotic foci were observed in the myometrium and one of which was connected to the membranous wall. The tumor is confined within the myometrium, not involved endometrium (Fig. 3). A myomatous nodule $(1 \times 1 \mathrm{~cm}$ in cross) was noted in the lower uterine segment. Surgical margin was free and there were no parametrial, lymphovascular and lymph node metastasis.

Microscopically, the tumor histologic type was CCA and there was no connection between the myometrial mass and endometrium and showed adenomyosis (Fig. 4). So, we identified that CCA arising from adenomyosis. The tumor was stained positively for p53 protein but negatively for estrogen receptor (ER) and progesterone receptor (PR). Based on these findings, this case was diagnosed as being CCA, FIGO (International Federation of Gynecology and Obstetrics) stage IB arising from adenomyosis after discussing with department of pathology. For personal reasons, she was transferred to other hospital and postoperatively whole pelvis radiotherapy and three cycles of systemic chemotherapy (paclitaxel $175 \mathrm{mg} / \mathrm{m}^{2}$ and carboplatin area under the curve 5) was performed to prevent local recurrence. Now she is healthy without any evidence of recurrence.

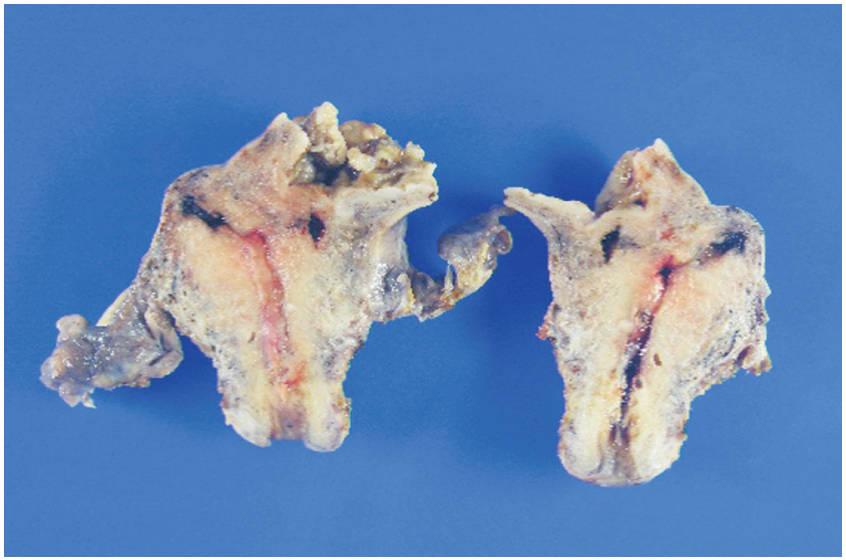

Fig. 3. Grossly, the uterus shows a papillary-surfaced solid tumor tissue surrounded by smooth membranous wall and several hemorrhagic foci in the myometrium. 

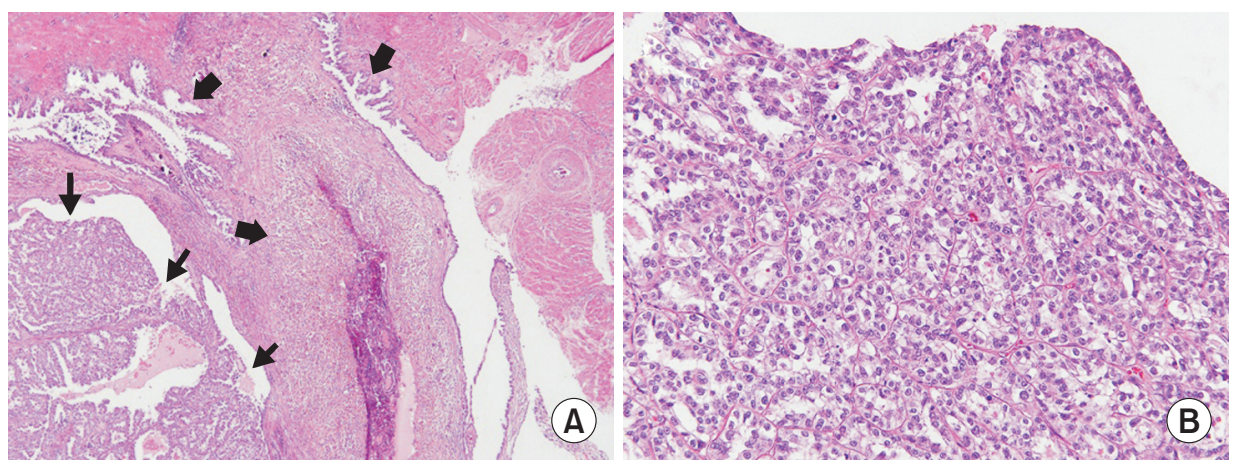

Fig. 4. Microscopically, Photomicrographs reveal (A) clear cell carcinoma (arrows) and adenymyosis (block arrows) in the surrounding myometrium and (B) abundant clear cytoplasm of the tumor cells.

\section{Discussion}

Malignant neoplasm arising from adenomyosis is very rarely encountered. The malignant transformation of patients with ovarian endometriosis has been reported approximately $0.7 \%$ to $1.0 \%$ [1]. Koshiyama et al. [2] reported that adenocarcinoma arising from adenomyosis comprises $0.7 \%$ of all uterine body carcinomas. Above all, CCA arising from adenomyosis has been very rarely reported [2,3]. Endometrioid adenocarcinoma is the most frequent histologic type of adenocarcinoma arising in endometriosis, followed by CCA [4]. In 1925, Sampson [5] introduced the histological criteria to define an adenocarcinoma arising in external endometriosis, which can be adapted to adenomyosis. Kumar and Anderson [6] emphasized the necessity of presence of the transition between the benign adenomyotic endometrial glands and the carcinomatous glands to prove the diagnosis of an ectopic endometrium-derived adenocarcinoma. Colman and Rosenthal [7] modified the diagnostic criteria by Sampson as follows: 1) the carcinoma should be absent in the normally situated endometrium and elsewhere in the pelvis; 2) the carcinoma should be actually observed to be arising from the epithelium of the areas of adenomyosis and not invading it from some other source; and 3) endometrial stromal cells should be surrounding the aberrant glands to support the diagnosis of adenomyosis. In our case, the diagnosis was compatible with CCA arising from adenomyosis because of the tumor being mainly located in the myometrium and identifying obvious transition between adenomyosis and CCA along with endometrial stromal cell. And the carcinoma was absent in the normally situated endometrium and elsewhere in the pelvis.

In general, the leiomyoma was positive for ER, PR, weakly positive for cyclooxygenase-2 and negative for P53 and CA 125
[8]. Hormone receptor expression is associated with low grade and early stage tumors. In contrast, an advanced cancer from the adenomyosis was reported as negative for ER and PR [9]. Ohta et al. [10] reported a case of a CCA arising from adenomyosis, with strongly expression of p53 and laminin-5 $\gamma 2$ chain. The poorly differentiated adenocarcinoma cells stained positively for p53, but did not express either ER or PR, suggesting that this adenocarcinoma was a type II carcinoma with biologically aggressive behavior. Due to the rarity, the characteristic feature of CCA arising from the adenomyosis is not well defined. However, we can expect that our patients will have a poor prognosis due to p53 positive and lack of ER and PR.

Baba et al. [11] suggested periodic follow-up using ultrasonography for early diagnosis and if any changes in mass consistency are suspected, an MRI should be performed for further evaluation. Malignant tumor arising from adenomyosis could be considered as a differential diagnosis when the patient with adenomyosis and intact endometrial surface.

In summary, we report a CCA patient arising from adenomyosis. It could have been misdiagnosed or diagnosed in delay because it was very rare and preoperative diagnosis was actually difficult. Therefore, when we find a cystic change with solid component in adenomyosis patients, CCA should be suspected as a differential diagnosis and MRI should be considered for further evaluation.

\section{Acknowledgments}

This research was supported by the 2018 scientific promotion program funded by Jeju National University. 


\section{References}

1. Irvin W, Pelkey T, Rice L, Andersen W. Endometrial stromal sarcoma of the vulva arising in extraovarian endometriosis: a case report and literature review. Gynecol Oncol 1998;71:313-316.

2. Koshiyama M, Suzuki A, Ozawa M, Fujita K, Sakakibara A, Kawamura M, et al. Adenocarcinomas arising from uterine adenomyosis: a report of four cases. Int J Gynecol Pathol 2002;21:239-245.

3. Hirabayashi K, Yasuda M, Kajiwara H, Nakamura N, Sato S, Nishijima Y, et al. Clear cell adenocarcinoma arising from adenomyosis. Int J Gynecol Pathol 2009;28:262-266.

4. Heaps JM, Nieberg RK, Berek JS. Malignant neoplasms arising in endometriosis. Obstet Gynecol 1990;75:1023-1028.

5. Sampson JA. Endometrial carcinoma of the ovary, arising in endometrial tissue of that organ. Arch Surg 1925;10:1-72.

6. Kumar D, Anderson W. Malignancy in endometriosis interna. $J$ Obstet Gynaecol Br Emp 1958;65:435-437.
7. Colman HI, Rosenthal AH. Carcinoma developing in areas of adenomyosis. Obstet Gynecol 1959;14:342-348.

8. Puppa G, Shozu M, Perin T, Nomura K, Gloghini A, Campagnutta $\mathrm{E}$, et al. Small primary adenocarcinoma in adenomyosis with nodal metastasis: a case report. BMC Cancer 2007;7:103.

9. Bergeron C, Amant F, Ferenczy A. Pathology and physiopathology of adenomyosis. Best Pract Res Clin Obstet Gynaecol 2006;20:511-521.

10. Ohta Y, Hamatani S, Suzuki T, Ikeda K, Kiyokawa K, Shiokawa A, et al. Clear cell adenocarcinoma arising from a giant cystic adenomyosis: a case report with immunohistochemical analysis of laminin-5 gamma2 chain and p53 overexpression. Pathol Res Pract 2008;204:677-682.

11. Baba A, Yamazoe S, Dogru M, Ogawa M, Takamatsu K, Miyauchi J. Clear cell adenocarcinoma arising from adenomyotic cyst: a case report and literature review. J Obstet Gynaecol Res 2016;42:217-223. 